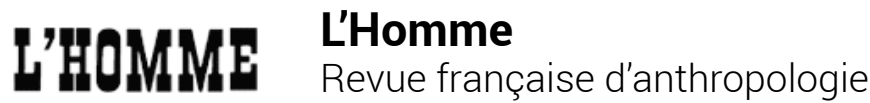

\section{Propriété privée et individu-sujet-de-droits}

La genèse historique de la notion de citoyenneté

\section{Colette Capitan}

\section{(2) OpenEdition}

1 Journals

Édition électronique

URL : http://journals.openedition.org//homme/4

DOI : 10.4000/lhomme.4

ISSN : 1953-8103

Éditeur

Éditions de l'EHESS

Édition imprimée

Date de publication : 1 janvier 2000

Pagination : 63-74

ISBN : 2-7132-1316-9

ISSN : 0439-4216

Référence électronique

Colette Capitan, « Propriété privée et individu-sujet-de-droits », L'Homme [En ligne], 153 I janvier-mars 2000, mis en ligne le 04 mai 2007, consulté le 19 avril 2019. URL : http://journals.openedition.org/ Ihomme/4 ; DOl : 10.4000/lhomme.4 


\section{Propriété privée et individu-sujet-de-droits \\ La genèse historique de la notion de citoyenneté}

\section{Colette Capitan}

P

OUR tenter de cerner les enjeux et les débats que soulève, aujourd'hui, la question du droit de citoyenneté, on ne peut partir d'une définition donnée pour acquise de celui-ci puisque cette question fait précisément l'objet du débat. En revanche, en observant les déterminismes politiques qui ont joué au cours de la Révolution française dans le sens d'une définition restrictive de l'exercice de ce droit, au moment, donc, où se forge une conception de l'entité nationale avec laquelle il se confond jusqu'à nos jours, on doit pouvoir en réactualiser le contenu.

Les principes fondamentaux qui ont sous-tendu l'histoire des démocraties occidentales des $\mathrm{XIX}^{\mathrm{e}}$ et $\mathrm{XX}^{\mathrm{e}}$ siècles - liberté, droits individuels, droits politiques, droit de citoyenneté, etc. - ne semblaient jamais devoir donner lieu à remise en question : ces acquis font si bien partie de la conscience moderne qu'ils semblent en avoir perdu leur caractère de conquête historique et avoir acquis un statut défiant l'histoire et le temps. Parmi ces acquis, la notion d'individu - le fait de s'appartenir soi-même - a généré plus qu'un système politique : un mode d'être, tant elle a informé historiquement et informe encore aujourd'hui quotidiennement notre manière d'être, de percevoir soi-même ou autrui, d'appréhender le monde matériel, de le penser, de le parler. Pour nous, le statut juridique de l'individu est clairement différencié de celui des biens matériels : une personne ne peut être directement ou indirectement l'objet d'une transaction marchande ou suivre le sort d'une transmission successorale par exemple, ni une propriété être le support de droits personnels ${ }^{1}$. 
Or, depuis peu, les débats que suscite la montée en puissance du toutéconomique semblent remettre ces certitudes en cause. Pour certains, l'État-nation n'apparaît plus comme le cadre propre à légitimer une société en voie de globalisation, l'autonomisation progressive de l'économie va permettre à celle-ci de se substituer au politique comme ressort premier et dernier de la société civile. Il s'agit, entend-on dire, d'étendre le marché à la totalité des relations sociales ${ }^{2}$. Dans ces conditions qu'en est-il de la citoyenneté, intrinsèquement liée au fait de la nationalité dans sa genèse et son histoire? Derrière cette revendication à l'effacement du politique et au tout-économique, faut-il voir se profiler un retour à un état social de non-droit? En d'autres termes, le statut de l'individu-sujetde-droits, contemporain et solidaire de la naissance des États-nations, estil voué à disparaître avec eux ?

Le succès même du modèle libéral a masqué l'histoire des circonstances qui ont amené à concevoir et à faire prévaloir, à l'aube des temps modernes, la notion du droit de propriété comme base de la liberté individuelle. Or, la théorie du droit naturel, que l'on crédite à juste titre de cette paternité, loin d'être homogène, a été en son temps l'objet de nombreuses controverses, dont l'une, majeure, a précisément porté sur la nature du lien entre propriété et liberté. Car si le lien entre le thème de la liberté et celui de la propriété représente un héritage venu du droit romain, commun à tous les auteurs, deux conceptions en émergent, aux conséquences radicalement contraires. Pour le dire schématiquement : les uns (Hugo Grotius, Samuel Pufendorf) entendent le droit de propriété comme un droit portant sur les choses et sur les individus, l'imperium (la souveraineté) se confond avec le dominium (le droit de propriété), la force est à l'origine du pouvoir institué comme le prouve le fait de l'esclavage ; les autres (John Locke et Jean-Jacques Rousseau) refusant de concevoir ce droit comme un droit à l'appropriation des personnes. Pour ces derniers, le droit de propriété, c'est le droit de s'appartenir soi-même, le droit à la vie, à la liberté : le droit sur les biens en est une conséquence. La force n'est pas à l'origine de la société, le contrat social, volontaire, est toujours révocable. Or, la Révolution française, seul exemple historique où l'on ait tenté d'appliquer dans les faits la théorie du droit naturel, adopte une troisième voie. Elle emprunte le principe (la liberté et les droits individuels sont fondés sur la propriété, le Contrat social est le signe commun d'appartenance à la nation, les droits politiques et la citoyenneté en dérivent) mais en conservant la part d'ombre héritée d'une tradition politique millénaire et

2. Ce vœu récemment exprimé sur une radio nationale ne visait pas, selon toutes apparences, à supprimer dans un proche avenir la part exorbitante du travail gratuit assuré dans le monde. 
devenue doctrine par l'intermédiaire de Rousseau : la mise à l'écart des femmes quant aux droits politiques. Cet interdit s'énonce au nom d'une idée de la nature qui n'a plus guère à voir avec celle d'un droit naturel valable pour tous mais il vient opportunément servir à instrumentaliser les femmes aux fins de la nation ${ }^{3}$. Ce faisant, l'option révolutionnaire renoue, par-delà les Lumières et le débat qu'elles avaient ouvert sur la part de l'acquis et de l'inné (l'éducation est-elle oui ou non responsable du statut inférieur des femmes dans la société ?), avec l'ambiguïté non résolue par la théorie du droit naturel elle-même, à l'aube de la modernité, quant à la nature de ces deux notions, la souveraineté et la propriété, et quant à leurs rapports réciproques.

Je traiterai successivement du syncrétisme propre au système prévalent antérieur à la société libérale - la société féodale - incarné dans le droit dit "de seigneurie» (le droit de seigneurie ne permet pas de distinguer "propriété animée " et "propriété non-animée »), de l'émancipation révolutionnaire/libérale de la notion de propriété, c'est-à-dire la dissociation des droits réels (relatifs aux choses) et des droits personnels (relatifs aux personnes), enfin de la théorie du droit naturel et de son acception française, à savoir la conception rousseauiste et révolutionnaire de la souveraineté nationale qui donne leur dimension politique à ces nouvelles catégories d'individu et de propriété.

\section{Le syncrétisme féodal}

Le système féodal se caractérise par la confusion du bien matériel avec la personne qui rend celui-ci « utile» ou "rentable» pour son seigneurpropriétaire $^{4}$. Dans un tel système, un bien non exploité est, au sens propre, sans valeur, une terre qui ne rapporte pas de droits «utiles» (qui

3. Une considérable réserve s'impose en effet concernant la thèse de la liberté chez J.-J. Rousseau pour peu qu'on fasse l'analyse non de ses seuls écrits classés politiques, mais celle de son œuvre dans sa totalité, et en particulier celle de l'Émile et dans l'Émile, le Livre V "Sophie ou la femme», rarement lu et jamais intégré à la lecture de l'ensemble : elle apporte un criant démenti à la thèse de la liberté si éloquemment plaidée par J.-J. Rousseau par ailleurs. La lecture de "Sophie ou la femme» relativise grandement le refus radical affiché par J.-J. Rousseau d'envisager la force à l'origine du contrat et de l'inégalité. En ce qui concerne le sort des femmes, J.-J. Rousseau plaide alors sans état d'âme la thèse de la force pure et simple d'un côté, de la soumission, contrainte et forcée au besoin, de l'autre. Le sous-titre de l'Émile, De l'éducation confirmerait s'il en était besoin la conviction de J.-J. Rousseau qu'on est bien, en ces matières, dans le social et non dans une présumée loi naturelle. Voir aussi, à ce sujet, les notes du Discours sur l'inégalité où J.-J. Rousseau démonte l'argumentation de John Locke tendant à justifier une relative inégalité naturelle des femmes.

4. Dans ce passage et le suivant, les termes ou expressions cités entre guillemets sont ceux du droit ancien. Dans la mesure où ils incarnent un système qui résiste efficacement et durablement - de 1789 à 1793 - aux reprises successives du travail législatif de l'abolition, ils sont omniprésents dans les esprits et les écrits de l'époque. 
rapportent quelque chose: une "rente», en travail, en produits, ou en argent) est, peut-on dire, sans coefficient social; elle est "vaine et vague». En fait - et en droit - la terre "utile » qualifie le statut seigneurial (que le «seigneur» puisse être un bourgeois ne change rien à l'affaire). Mais la terre n'est "utile" que pour autant qu'un ou des moyens d'exploitation y sont attachés pour la rentabiliser. Dans l'Ancien Régime le paysan "suit " le statut de la terre : même «libre », il est assujetti aux "droits féodaux».

À la fin du XVIII e siècle, l'Ancien Régime représente un assemblage confus de droits issus de deux régimes distincts à l'origine : le plus ancien, le «système seigneurial », qui réfere aux notions de "souveraineté » (du seigneur) et de dépendance ; et le système «féodal » proprement dit qui réfere aux notions de "propriété éminente " (du suzerain) et de "vassalité ». À la veille de la Révolution, l' "Ancien Régime" a fait flèche de tout bois, les deux systèmes ont fusionné pour n'en faire plus qu'un aux effets cumulés : il a intégré le principe de propriété hérité du système féodal et celui de souveraineté issu du système seigneurial, amalgamé l'appropriation vassalique du système féodal déclinant à la souveraineté seigneuriale croissante, réduit la vassalité à la dépendance.

Un tel système ne permet pas de distinguer les biens matériels des biens non matériels, la terre de l'instrument : outils, bétail ou paysan, qui la rendent «utile». La perception qu'on a de ceux-ci passe par la médiation de celle de la terre. Terre et paysans forment une entité, factuelle et juridique. Ils ne sont pas perçus comme hétérogènes mais comme solidaires, dans leur seul rapport au maître-propriétaire. Celui-ci est investi de l'ensemble des prérogatives de la puissance publique et du droit à l'appropriation sur la nature venus des systèmes antérieurs : le "droit de seigneurie ». L'asservissement du paysan à la terre assure le statut domanial du seigneur, qui à son tour rejaillit sur le paysan. Clef de voûte de l'ensemble, le droit de seigneurie incarne donc un système clos, tautologique, de pratiques et de représentations où êtres et biens se définissent mutuellement et réciproquement, où il n'y a pas plus de terres sans paysans qu'il n'y a de paysans sans terre. Un système qui ne permet pas de distinguer "propriété animée " de "propriété non animée " ${ }^{5}$, de conceptualiser l'individu humain distinctement du bien matériel. C'est ce syncrétisme qui qualifie la "féodalité » à la veille de 1789 : l'individu humain, a fortiori un individu sujet de droits, y est un impensé politique, car structurellement impensable.

5. J'emprunte ici un peu librement la terminologie de Claire Michard. Voir C. Michard, «Approche matérialiste de la sémantique du genre en français contemporain ", in Marie-Claude Hurtig, Michèle Kail \& Hélène Rouch, eds, Sexe et genre : de la hiérarchie entre les sexes, Paris, Éditions du CNRS, 1991 : 147-157. 


\section{La distinction des "droits réels" et des "droits personnels"}

Pour que la circulation et l'échange des personnes, des biens et des idées puissent s'opérer, pour passer à une société libre, il fallait rompre ces liens caractéristiques de la "féodalité", autrement dit, il fallait pouvoir concevoir hommes et choses, hommes et biens, comme distincts. Pour cela, la seule Déclaration initiale d'abolition du "système féodal» (en 1789) ne pouvait suffire. La Déclaration posait un principe, solennel et révolutionnaire, mais insuffisant à lui seul pour entraîner des effets concrets immédiats. Tout le travail révolutionnaire tient donc à cet effort qui, partant d'un principe volontariste théorique, a consisté à intégrer progressivement les leçons tirées de la résistance des faits - de la résistance surtout de l'ancienne classe dominante, toute prête à endosser pour elle-même les bénéfices de cette progressive émancipation foncière. Au prix d'un long travail de va-et-vient entre éclaircissements d'ordre conceptuel et démentis opposés par les faits, les révolutionnaires devaient arriver à concevoir la notion d'un droit de propriété privée suffisant pour fonder une économie libérale de marché. Lorsque les droits purement fonciers émergent en tant que droits clairement distincts des droits «féodaux " (juillet 1793), l'individusujet-de-droits devient réalité politique.

Il convient de s'arrêter un instant sur le travail qu'a représenté l'abolition d'une redevance caractéristique de l'" ancien système", celle du "cens». L'analyse du cens est en effet décisive, du fait de son exemplarité quant à la confusion et aux enjeux des différents droits évoqués ci-dessus. Et c'est aussi le cens de toutes les redevances personnelles ou réelles pesant sur la terre qui donna le plus de fil à retordre aux révolutionnaires.

À l'origine, le cens était une redevance "féodale», connotant la "propriété éminente" du suzerain. Vite tombée comme les autres redevances féodales sous la coupe du régime seigneurial de la "dépendance ", le cens, une redevance en argent, est "récognitif de la directe seigneuriale». À la fin du XVIII siècle, la "censive" (ou "tenure roturière", "louée à cens") était le type d'exploitation le plus répandu. La censive était-elle une propriété paysanne, ou pas? La question a longtemps divisé les historiens. Si le cens avait été une survivance toute symbolique d'un système "révolu " dans les faits, comme tant d'historiens nous l'assurent ${ }^{6}$, il aurait dû témoigner de la réalité d'une propriété paysanne dès les premières mesures

6. En particulier François Furet dans Penser la Révolution française, Paris, Gallimard (« Bibliothèque des histoires »), 1978. 
d'abolition. Or, on est loin du compte et le cens survit durablement à tous les décrets : à l'abolition de la « servitude personnelle » (1789), à l'abolition du caractère "féodal» des redevances (1790), et même au décret qui déclare la propriété foncière " franche et libre de tous droits» (1792). C'est la suppression, par la Convention (première assemblée élue), de «la présomption du titre primitif de propriété" (ou présomption d' «inféodation») (1793) qui, en émancipant définitivement la propriété paysanne, révèle la véritable nature du cens maintenu jusque-là : celle d'une redevance de servitude. Aboli révolutionnairement comme marque de servitude, le cens survit politiquement au-delà pour distinguer les «citoyens actifs » des " citoyens passifs » ${ }^{7}$.

La propriété, condition de la liberté ${ }^{8}$. Pour les révolutionnaires, la propriété ne pouvait pas ne pas matérialiser la liberté: dans le "système ancien ", la terre est le signe de la servitude, elle sera le signe de la liberté. De la propriété dérivent directement identité individuelle, droits juridiques, droits politiques. Selon les constitutions révolutionnaires successives, "l'homme libre est celui qui ne dépend d'aucun autre, qui n’appartient à personne ». D’où le caractère "sacré » de la propriété privée révolutionnaire: elle est le fruit de "l'industrie» (i.e. du travail) dont dépend la liberté. L'émancipation de la nation (territoire et nouvelle entité politique) fait du nouveau pouvoir légitimant l'héritier désigné de l'ancienne classe dominante, elle garantit "souverainement " propriété privée et libertés individuelles.

Par les contraintes spécifiques qu'elle eut à vaincre, mais aussi par les visées qui étaient les siennes - ouvrir à l'économie de marché - l'émancipation libérale soulève donc une série de questions. La première tient à la nature et à l'extension du droit de propriété ainsi défini. Quant à son extension: les révolutionnaires ont procédé à l'émancipation des biens pour autant que celle-ci était nécessaire et suffisante au développement d'une économie de marché, à la libre disposition, à l'échange et à la circulation, de biens. Quant à la nature de ce droit, sa définition, utilitaire, laisse irrésolu le problème de l'amalgame entre le principe de souveraineté et celui de la propriété : l'analyse du système confondant patrimonialité et souveraineté reste à faire.

L'ambiguïté qu'entraîne cette analyse inachevée du «droit de seigneurie » quant aux droits et au statut des personnes est patente. Pour les « asso-

7. Pour plus de détails, je renvoie à Colette Capitan, La nature à l'ordre du jour, 1789-1793, Paris, Kimé, 1993.

8. Décret du 20 août 1792 : «L'Assemblée nationale, considérant que l'affranchissement des propriétés, en assurant l'indépendance absolue des citoyens, peut seul leur procurer la jouissance pleine et entière de la liberté $[\ldots]$ décrète...» 
ciés de la grande entreprise sociale " (la formule est de Sieyès) le droit de propriété ainsi dégagé implique ipso facto droits politiques et citoyenneté : la propriété est incontestablement la condition de la liberté. Mais pour les autres? Qu'en est-il des individus "sans ressources " : indigents, étrangers, femmes, mineurs..., bref de tous ceux qui ne sont pas conviés à la signature du nouveau contrat social, qui ne sont pas "associés [à] l'entreprise " ? Privés de droits, ils se trouvent dans une zone mal définie juridiquement, maintenus dans une situation objective de dépendance et d'infériorité, pas vraiment individus-à-part-entière, et certainement pas "citoyens ». Le vote, aux tous débuts de la Révolution, des décrets instituant l'« argent-marchandise » ouvre symboliquement l'ère du tout-économique. Ne peut-on considérer qu'à la difficulté qu'a eu le droit naturel de penser le statut des femmes dans la société politique, s'ajoute alors une ambiguïté supplémentaire, la menace d'une objectivation de tous les laissés-pour-compte de l'émancipation ${ }^{9}$ ?

\section{Droit naturel, contrat social et souveraineté nationale}

La théorie du droit naturel n'a pas de représentants en France et si la doctrine elle-même est bien connue, c'est moins aux jurisconsultes allemands (Hugo Grotius, Samuel Pufendorf) qu'aux auteurs politiques (Thomas Hobbes, John Locke) qu'elle le doit ${ }^{10}$. Lorsque les philosophes, et Rousseau plus spécialement, renouent avec la théorie, ce n'est pas sans y introduire nombre de distorsions. La question, au départ, est la même pour tous les auteurs: y a-t-il à l'origine des sociétés humaines une loi naturelle, édictant des règles universelles ? Si tel est le cas, en quoi consiste cette loi ? Quels rapports y a-t-il entre le droit naturel et le droit positif ? C'est tout le débat sur la liberté et la nature humaine qui est ici en jeu.

Que disent à ce sujet Locke et Rousseau, l'auteur fétiche des révolutionnaires ${ }^{11}$ ? Chez Locke, la property réfere non aux biens matériels, mais au fait

9. L'institution de l'«argent-marchandise " (1790) a été le fer de lance de la révolution populaire contre la révolution bourgeoise. Tout au long de l'An II, adresses et pétitions affluent à la Convention pour dénoncer «la nouvelle aristocratie " qui se met en place grâce à elle. Mais au-delà ? La vraie question ne serait-elle pas celle du statut que cette nouvelle inégalité induit pour les non-propriétaires, c'est-à-dire pour les non-citoyens en l'occurrence? Pour ces derniers (étrangers, domestiques, femmes), revendiquer l'un quelconque des signes qui sont les attributs de la citoyenneté: le patriotisme, la réussite dans les affaires, politiques ou non, les honneurs, les fonctions, les privilèges, les met en situation objective de suspects. Ce qui joue en faveur des citoyens joue en défaveur de ceux qui ne le sont pas.

10. Hugues Grotius, Le droit de la guerre et de la paix, nouvelle trad. du latin par Jean Barbeyrac, Amsterdam, P. de Coup, 1724 (1625); Samuel Pufendorf, Le droit de la nature et des gens, trad. du latin par Jean Barbeyrac, Amsterdam, H. Schelte et J. Kuyper, 1706 (1672); Thomas Hobbes, De Cive, Parisiis, 1642 ; John Locke, Two Treatises of Government, edited with an introduction and notes by P. Laslett, Cambridge, 1990 (1690). [Trad. fr. Essai sur le pouvoir civil de John Locke, Paris, PUF, 1953.]

11. Les révolutionnaires se sont davantage réclamés de Locke que de Hobbes. Rousseau emprunte à l'un et à l'autre au moins autant qu'il s'en démarque, mais il s'oppose nettement à Hobbes sur quelques-uns .../... 
de s'appartenir soi-même, une notion héritée de la tradition de l'Habeas corpus: droit à la vie, à l'intégrité de son corps et de sa personne, aux fruits de son travail, etc. Tous droits dont dérive le droit d'appropriation sur la nature, comme fruit de son industrie. Le droit de propriété n'est pas la condition de la liberté, il en est l'effet. Et, de ce point de vue, tous les êtres, femmes ou hommes, sont égaux : la société civile ou politique repose sur la raison, tous les êtres humains en étant normalement pourvus, tous participent du contrat social. La société est faite pour défendre la vie et la propriété de soimême, aider au développement et au plein épanouissement des facultés de chacun. Pour Locke, société civile et société politique étant une seule et même chose, le contrat est toujours révisable, toujours virtuellement extensible au profit d'un plus grand nombre de signataires ${ }^{12}$.

Rousseau partage avec Locke la conviction intime que la liberté est un droit qui ne s'aliène pas ${ }^{13}$. Une conception de la liberté qui sera aussi celle des révolutionnaires ("Tout homme peut engager ses services, son temps; mais il ne peut se vendre, ni être vendu; sa personne n'est pas une propriété aliénable... ", art. 18 de la Déclaration des droits de l'homme et du citoyen de 1793). Mais il ne conçoit pas cette liberté comme étant de droit universel : il explique le social par le social ${ }^{14}$, son approche est d'ordre politique. Pour Rousseau, la société naturelle n'existe pas, c'est une fiction - tout comme l'est d'ailleurs le contrat postulé à l'origine de la société humaine - dont le caractère, à la fois utopique et artificiel, sert tout au plus d'argument à la démonstration a contrario. S'il recourt à ce schéma, traditionnel dans le débat, c'est pour lui pure commodité intellectuelle. Pour Rousseau, ce n’est pas la force ( «la force n’est rien sans le droit»), c'est le droit qui fonde les sociétés humaines. L'homme n'est véritablement homme, dit-il dans la première version du Contrat (dit Manuscrit de Genève), que parce qu'il est d'abord citoyen, et c'est cette conviction qui donne sa force et confere ce ton de modernité à sa pensée. La convention qu'il imagine à l'origine des sociétés humaines lui est le moyen propre à développer, en dépit de ou grâce à ce caractère d'artifice, une conception de la société humaine qui est, avant tout autre commentaire qu'on en puisse faire, une conception purement sociale de la société.

\footnotetext{
des points qui nous intéressent plus particulièrement ici : en particulier sur la question de l'état de nature (pure fiction intellectuelle pour Rousseau) et sur la définition de la souveraineté. Pour une analyse détaillée des rapports entre J.-J. Rousseau et T. Hobbes, voir R. Derathé, Jean-Jacques Rousseau et la science politique de son temps, Paris, Vrin, 1995.

12. John Locke, Two Treatises of Government, op. cit., 1990.

13. Jean-Jacques Rousseau, Discours sur les origines et les fondements de l'inégalité parmi les hommes, Paris, Gallimard ( "Bibliothèque de la Pléiade» III), 1964 (1754); Du Contrat social, in ibid. (1762).

14. À la suite d'Émile Durkheim, Claude Lévi-Strauss a depuis longtemps reconnu le sociologue en Rousseau, cf. Tristes tropiques, Paris, Plon, 1955.
} 
Chez Rousseau, le contrat social est le signe, la marque même de la dimension sociale de l'homme.

Comme Locke, Rousseau est convaincu que l'homme réalise son plein développement (intellectuel, sensible, physique) à l'intérieur d'une communauté choisie et acceptée. Par elle, il renonce à une supposée liberté originelle, qui ne peut être que la liberté du non-droit : les avantages de l'état civil sont tels « que si les abus de cette nouvelle condition ne le dégradaient souvent au-dessous de celle dont il est sorti, il devrait bénir sans cesse l'état heureux qui l'en arracha pour jamais et qui, d'un animal stupide et borné, fit un être intelligent et un homme" (Manuscrit de Genève). "L'homme est né libre ", la liberté est une conséquence de la nature de l'homme, et la nature de l'homme est d'être social. L'homme est perfectible, il est perfectible parce qu'il est un être social, et il n'a pas plus le droit d'aliéner sa liberté, qu'il ne peut consentir à en être privé.

Ce thème de l'aliénation, qu'il est l'un des premiers à esquisser, témoigne à lui seul de la conviction de Rousseau que l'inégalité est bien l'effet du droit. Il serait sans fondement dans l'hypothèse de la force à l'origine des sociétés. Rousseau récuse la thèse dominante des théoriciens du droit naturel (Grotius, Hobbes) selon laquelle la force serait à l'origine des sociétés comme il récuse l'argument de fait invoqué par Grotius, le cas de l'esclavage : «S'il y a [...] des esclaves par nature, c'est parce qu'il y a eu des esclaves contre nature", dit-il. Pour Grotius ou Pufendorf, l'idée d'un droit inaliénable est impensable. Pour Rousseau au contraire, le caractère inaliénable de la liberté individuelle, comme celui de la souveraineté, est le point absolu d'où tout découle ${ }^{15}$. On ne peut aliéner, disposer, vendre ou acquérir qu'en vertu d'un droit qui n'apparaît qu'au sein de la société civile, qu'en vertu d'un droit qui doit son origine à des conventions humaines. Admettre qu'on puisse aliéner sa liberté serait admettre, dit-il, l'aliénation de l'homme dès son origine, chose impensable car «l'homme est naturellement son propre maître ». La liberté est un droit naturel et il est de l'essence d'un tel droit d'être inaliénable.

Ce parti-pris d'argumenter sur le fondement des sociétés humaines «en raison » et son refus d'envisager un état ante-social à ce qui fait précisément le caractère spécifique de l'humanité sont, il faut le répéter, tout à fait remarquables de sa part. Aussi, quand Rousseau exclut les femmes du contrat en tant qu'agents actifs, quand il reprend, pour la circonstance, le thème de la nature pour argumenter cette exclusion (thème absent de ses

15. Pour J.-J. Rousseau, le Souverain, personne morale qui s'incarne dans la volonté générale, est à l'image de l'individu : comme la liberté, la souveraineté est inaliénable et indivisible. 
œuvres dites politiques), cela indique assez la nature politique du projet ${ }^{16}$. Son plaidoyer en faveur de la liberté et de son inaliénabilité milite en sa faveur et répond par avance au grief qu'on lui a souvent fait, de totalitarisme: sa théorie de l'État est une théorie contractuelle, même s'il ne reprend pas dans la version définitive du Contrat la thèse de l'insubordination légitime développée dans le Manuscrit de Genève. Mais il faut bien admettre que ce plaidoyer se trouve radicalement infirmé dès lors qu'il s'agit d'en tester l'universalité : la liberté, pour Rousseau, ne se conçoit que comme le privilège d'une part, et d'une part seulement du social. Et si, comme il le pense, "la liberté politique est la garantie de toutes les autres », alors l'idée de nature devient, par ses soins, l'arme idéologique absolue de la domination ${ }^{17}$.

Tous les philosophes des Lumières partagent peu ou prou la même problématique de la nature. Mais c'est à Rousseau qu'on doit, sinon le glissement que subit la théorie du droit naturel lors de son passage en France - celui qui va de l'idée d'un droit naturel à la liberté individuelle à l'idée d'une loi naturelle justifiant la domination - du moins l'argumentaire, et son extraordinaire succès, d'une loi de nature censée déterminer la seule classe de sexe des femmes. Leur privation des droits politiques par la Révolution, leur réduction à un statut minoritaire, c'est à Rousseau qu'elles le doivent, Rousseau, le doctrinaire et le vulgarisateur de l'enfermement des femmes dans l'idéologie naturaliste. Autant son discours de la liberté, de la perfectabilité humaine convainc de la nature sociale de l'individuation, autant celui de la force pure et simple, de la contrainte, de l'humiliation et de l'abêtissement nécessaires des femmes, qu'il théorise, fait désespérer de la première.

L'amour de l'ordre que J.-J. Rousseau exprime à la fin du Contrat («l'ordre et la paix », dit-il, sont un "droit sacré»-Manuscrit de Genève), son brusque ralliement à l'état de choses, qui le ramène à l'argument de fait invoqué par Hugo Grotius et qu'il rejetait au départ, l'éloge qu'il fait de la Loi, n'ont pas d'autre justification : tout défectueux qu'il soit, le contrat a le

16. Au XVIII ${ }^{\mathrm{e}}$ siècle, la traduction de civitas est société civile. J.-J. Rousseau emploie le terme dans le Discours sur l'inégalité, il y renonce dans le Contrat, non sans avoir hésité. Il est en effet intéressant de noter que, sous l'influence de John Locke (pour qui political society égale civil society), Rousseau a hésité sur le titre de la première version du Contrat qu'il a été tenté d'intituler "De la société civile». Voir Derathé 1995, op.cit. : Appendice.

17. La désignation par le terme d'universel du principe selon lequel il existe un droit, pour tout être humain, de prendre part aux affaires publiques est aujourd'hui remis en question du fait de son échec historique. À ceci, on peut toujours objecter que ce n'est pas parce qu'un principe a été détourné ou confisqué au départ qu’il est pour autant invalidé. En revanche, on peut craindre que le terme de "parité " proposé en lieu et place de celui d'universalité ouvre la porte à d'autres dérives autrement redoutables par le risque qu'elle induit d'une naturalisation codifiée des sexes. Entre l'échec historique d'un principe qui, à l'analyse, garde toute sa charge révolutionnaire et la naturalisation des groupes sociaux, il faut choisir. 
mérite de légitimer l'ordre naturel des choses, le fait établi de l'inégalité entre les femmes et les hommes, un fait établi qui se confond pour J.-J. Rousseau avec le Bien. Car "à considérer humainement les choses", les lois humaines sont imparfaites. Elles n'en sont pas moins justes puisque, en fin de compte, " ce qui est bien et conforme à l'ordre est tel par la nature des choses et indépendamment des conventions humaines".

À l'issue de la Révolution, le bilan est donc pour le moins contrasté. Le XVIII ${ }^{e}$ siècle a rouvert le débat sur la nature, la liberté et l'aliénation de l'homme, la Révolution ouvert celui sur les rapports de pouvoir et le droit à l'appropriation ou à l'asservissement d'autrui : la mise à l'écart des femmes du débat public est le révélateur de la définition de la citoyenneté dont nous avons hérité, d'une part, et du statut réservé aux exclus de l'émancipation, de l'autre.

C'est contraints et forcés que les révolutionnaires ont pris acte du fait que l'émancipation des individus, pour se concrétiser, passait par celle, prioritaire car déterminante, de la propriété ${ }^{18}$. Ce préalable d'ordre matériel à l'exercice des droits politiques - la terre ou l'argent ${ }^{19}$ - induit la définition de la citoyenneté : le droit de propriété est le fruit de "l'industrie", le travail le signe de la liberté et de l'autonomie ; le "droit au sol », le plus court chemin pour signifier la victoire révolutionnaire sur «l'ancien système ", confere donc les droits citoyens ${ }^{20}$.

Pour tous ceux qui sont sans ressources, qui n'ont pas la libre disposition d'eux-mêmes, quel qu'en soit le motif (incapacité, chômage, dépendance), s'ajoute à leur exclusion le risque d'une instrumentalisation aux fins de la nation. La trilogie révolutionnaire - propriété, autonomie, droits politiques - n'est pas nécessairement périmée. Elle est à réviser dans ses priorités et à conformer aux visées initiales des Lumières qui avaient compris la leçon de Locke pour qui la participation à la vie publique donne sa pleine mesure à la condition humaine.

MOTS CLÉS/KEYWORDS : citoyennetélcitizenship - droit naturel/natural law - droit de propriété/feudal law - souveraineté/sovereignty - Lumières/Enlightenment.

18. De même, l'émancipation collective, celle de la nation, passe par celle du territoire national.

19. L'institution de "l'argent-marchandise " a précédé la mise en place des institutions politiques révolutionnaires: le même mécanisme se répète aujourd'hui avec la mise en place de l'Europe monétaire. 20. L'individu est-il le produit d'une copulation ou celui de l'individuation sociale ? Les débats, aujourd'hui, sur le statut ontologique de l'individu humain (à propos de l'avortement, des manipulations génétiques, du clonage...) montrent la permanence d'une définition de l'individu à partir de sa seule physicalité. 
Colette Capitan, Propriété privée et individusujet-de-droit. Le genèse historique de la notion de citoyenneté. - Le droit de citoyenneté est un acquis révolutionnaire de la fin du siècle des Lumières. Historiquement lié à la genèse du fait national, il ne s'y réduit pas. Pour les ténors du droit naturel (Locke, Rousseau), l'exercice des droits politiques parachève le droit à la liberté et à la propriété de soimême, il consacre la victoire de l'émancipation individuelle et collective sur l'arbitraire du souverain. Réserver ou interdire l'exercice du droit de citoyenneté revient à pérenniser le droit «féodal " qui confond droit de souveraineté et droit d'appropriation sur les personnes et les biens.
Colette Capitan, Private Property and the Individual in Law: The Historical Genesis of the Notion of Citizenship. - Acquired through a revolution at the end of the Enlightenment, the right of citizenship cannot be reduced to the genesis of the nation-state, with which it is associated. For the advocates of natural law (Locke, Rousseau), the exercise of political rights crowned the right to freedom and to the "property" of one's self. To restrict or forbid the exercise of citizenship amounted to perpetuating feudal law, which confused the right of sovereignty with the right to own persons and goods. 